\title{
Neurotropic Effect of Carbamylated Darbepoietin on the Model of Primary Hippocampal Culture
}

\author{
DOI: $10.17691 / \mathrm{stm} 2019.11 .4 .10$
}

Received March 3, 2019

\begin{abstract}
O.M. Shirokova, Junior Researcher, Electron Microscopy Department, Institute of Fundamental Medicine 1 ;
R.A. Sokolov, Junior Researcher, Center for Translational Research;

S.A. Korotchenko, Researcher, Molecular and Cellular Technologies Department, Institute of Fundamental Medicine ${ }^{1 ;}$ V.I. Pershin, PhD Student, Department of Neurotechnologies, Institute of Biology and Biomedicine2; Laboratory Assistant, Molecular and Cellular Technologies Department, Institute of Fundamental Medicine; K.V. Ermin, Junior Researcher, Molecular and Cellular Technologies Department, Institute of Fundamental Medicine";

M.M. Glyavina, PhD Student, Department of Neurotechnologies, Institute of Biology and Biomedicine ${ }^{2}$; Laboratory Assistant, Morphology Department, Institute of Fundamental Medicine';

M.A. Zhuchenko, PhD, Chief of Section;

N.N. Andreeva, DSc, Professor, Department of Normal Physiology named after N.Y. Belenkov';

N.A. Shchelchkova, PhD, Associate Professor, Department of Normal Physiology named after N.Y. Belenkov';

Associate Professor, Department of Neurotechnologies, Institute of Biology and Biomedicine; Head of Molecular and Cellular Technologies Department, Institute of Fundamental Medicine'; I.V. Mukhina, DSc, Professor, Director of the Institute of Fundamental Medicine ${ }^{1 ;}$;

Head of the Department of Normal Physiology named after N.Y. Belenkov1; Professor,

Department of Neurotechnologies, Institute of Biology and Biomedicine ${ }^{2}$

${ }^{1}$ Privolzhsky Research Medical University, 10/1 Minin and Pozharsky Square, Nizhny Novgorod, 603005, Russia; ${ }^{2}$ National Research Lobachevsky State University of Nizhni Novgorod, 23 Prospekt Gagarina, Nizhny Novgorod, 603950, Russia;

3LLC "FARMAPARK", 8/1 Nauchny proyezd, Moscow, 117246, Russia
\end{abstract}

Activation of the cerebral erythropoietin system can be a promising strategy for the management of various neurodegenerative and neuropsychiatric diseases as it triggers neuroprotective mechanisms and improves cognitive functions. Lack of information about the possible neurotrophic effect of erythropoietin reduces the possibility of using the brain receptor for erythropoietin as a therapeutic target in neurodegenerative diseases associated with hypoxia and inflammation.

The investigation aims to study the influence of the erythropoietin receptor agonist - carbamylated darbepoetin (CdEpo) - on the morphofunctional features of neuron-glia networks of primary hippocampal cultures in mice under normoxia.

Materials and Methods. Primary hippocampal cell cultures dissociated from embryos (E18) of C57BL/6 mice were used to study the influence of erythropoietin receptor stimulation on the functional activity of hippocampal neuron-glia networks. The experiments were carried out on days 18-23 of culture development in vitro. CdEpo $(100 \mathrm{ng} / \mathrm{ml})$ action duration was $24 \mathrm{~h}$. Functional changes were assessed based on the electrical and metabolic activity of cultured cells using patch-clamp techniques, multielectrode registration of bioelectric activity in neural networks and calcium imaging, respectively. The morphological features of primary hippocampal cell cultures were studied using transmission electron microscopy.

Results. Treatment of primary hippocampal cell cultures with CdEpo during $24 \mathrm{~h}$ did not affect the frequency of spontaneous single action potentials, spontaneous burst activity of cells, the patterns of neuron action potentials (the amplitude of depolarization phase, threshold potential, the amplitude of hyperpolarization phase), membrane capacity. It did not affect the bioelectric parameters of neural network activity (the number of spikes in a network burst, network burst duration, and inter-burst interval), calcium activity of neurons and glial cells determined by the duration and frequency parameters of spontaneous calcium oscillations. At the ultrastructural level, the number of mature asymmetric synaptic contacts remained unchanged under the influence of CdEpo, but there was a morphogenesis of the internal structure of dendritic spines: the number of spines with endoplasmic reticulum and/or the spine apparatus inside increased, which was a unique phenomenon for a model of primary hippocampal culture.

Conclusion. There was revealed no neurotropic effect of CdEpo on the model of primary hippocampal culture as reflected by bioelectric activity parameters of single neurons, neuronal networks, and astrocytic network activity evident in the parameters of intracellular calcium concentration changes (calcium oscillations) under normoxia conditions. However, CdEpo causes changes in the internal structure of dendritic spines in some neurons with the spine apparatus appearing in them. The absence of CdEpo effect on the functions of intact neurons and glia indicates the relative safety of using this molecule for therapeutic purposes as cytoprotection for the brain tissue.

Key words: erythropoietin receptor; hippocampus; primary hippocampal cultures; neuron-glia networks; neuronal ultrastructure; spine apparatus; patch-clamp technique; calcium imaging.

Corresponding author: Olesya M. Shirokova, e-mail: shirokovaom@gmail.com 


\section{Introduction}

Activation of the erythropoietin system of the brain can be a promising strategy for the management of various neurodegenerative and neuropsychiatric diseases as it triggers neuroprotective mechanisms and improves cognitive functions [1, 2]. It has been found that erythropoietin released by astrocytes participates in erythropoiesis activation and stimulates migration of neuroblasts to the area of ischemic brain damage, i.e. nervous tissue regeneration [3]. The transcription factor triggering erythropoietin gene expression in astrocytes in hypoxia appeared to be hypoxia-inducible factor 2 (HIF-2) [4].

The neuroprotective effect of erythropoietin is supposed to be mediated by a heterocomplex of heterodimeric tissue-protective receptor and $\beta$-common receptor subunit ( $\beta c R$, or $C D 131)$ [5], which is structurally different from the classical homodimeric erythropoietin receptor (EpoR) involved in erythropoiesis [6]. Besides, short-term ligand access to the heterodimeric receptor is sufficient to trigger the neuroprotection mechanism, whereas stimulation of erythropoiesis with homodimer EpoR requires long-term ligand influence [7]. The $\beta c R$ protein belongs to type 1 cytokine receptor subfamily and can form heteromeric receptors with IL-3, IL-5, and GM-CSF receptor subunits. $\beta c R$ integration into the cell membrane was found to change in response to cellular stress [8]. The difficulty of studying erythropoietin receptors in the brain is caused by lack of reliable commercial antibodies to the heterodimer receptor complex $[9,10]$. At present, molecular mechanisms of erythropoietin action and its derivatives in the brain remain unclear. In particular, there is no data on their possible neurotrophic effect, which reduces the possibility of using the brain receptor for erythropoietin as a therapeutic target in neurodegenerative diseases, including those associated with hypoxia and inflammation.

In our study, a carbamylated form of darbepoetin (CdEpo) is proposed as an agonist to the receptor heterodimer. CdEpo is obtained by carbamylation of all amino acid residues of lysine included in the darbepoetin molecule and the alanine residue in the $\mathrm{N}$-terminal region of the protein. Erythropoietin carbamylation leads to modification of the molecule sites binding to the EpoR receptor, in particular, the high-affinity site Pro42-Trp51 containing lysine residue Lys45. Loss of hematopoietic activity is likely to result from carbamylation of this residue [11].

Since the use of the agonist of heterodimeric erythropoietin receptor as a neuroprotector involves interaction with nervous system cells, it is necessary to evaluate the possibilities of CdEpo to produce a neurotrophic effect. In this regard, the investigation aims to study the effect of carbamylated darbepoetin on the morphofunctional features of neuron-glia networks of cells in the model of primary culture of mouse hippocampus.

\section{Materials and Methods}

Primary hippocampal cultures. The study focuses on primary hippocampal cells obtained from 18-dayold embryos of C57BL/6 (E18) mice and cultured for 23 days. Care and management of experimental animals complied with the norms provided in the Order of the Ministry of Health of the Russian Federation No.199n dated April 1, 2016 "Approval of the Rules for Good Laboratory Practice", the National Standard of the Russian Federation (GOST 33044-2014 "Principles of Good Laboratory Practice"), sanitary and epidemiological regulations 2.2.1.3218-14 No.51 dated August 29, 2014. The work was approved by the Ethics Committee of Privolzhsky Research Medical University.

Cells were dissociated by treating the hippocampal tissue with $0.25 \%$ trypsin (25200-056; Invitrogen, USA). The culture environment was changed every other day. The viability of the cultures was maintained in a $\mathrm{CO}_{2}$ incubator at $35.5^{\circ} \mathrm{C}$ in a gas mixture containing $5 \%$ $\mathrm{CO}_{2}$. Cell cultures were taken for studies on days 18 23 of their development in vitro. Preliminary electron microscopic studies showed [12] that neurons interacted in the network mainly through mature synaptic contacts at that stage of primary culture development. The functional activity of neuron-glia networks was estimated $24 \mathrm{~h}$ after adding carbamylated darbepoetin $(100 \mathrm{ng} / \mathrm{ml})$ to the culture medium.

Calcium imaging. Functional activity of neurons and glia was monitored based on short-term changes in intracellular calcium concentration (calcium oscillations) using fluorescence microscopy with LSM 510 confocal laser scanning microscope (Carl Zeiss, Germany). Oregon Green 488 BAPTA-1 AM (Thermo Fisher Scientific, USA) was used as a calcium probe. Recordings of calcium oscillations (events) lasted 10 min with image frequency of $4 \mathrm{~s}^{-1}$. The resulting series of images were processed using the original software [13]. The software provides the possibility to build for each cell the graphs of fluorescence intensity dependence on time and mark the oscillatory events, it also allows determining such parameters as frequency and duration of calcium events.

Electrical activity. Electrophysiological parameters of single neurons in the networks of primary cultures of mouse hippocampus were determined using the patchclamp technique in the whole-cell configuration. For this purpose, the following equipment was used: the SliceScope Pro 2000 device (Scientifica, UK), EPC 10 USB double patch-clamp amplifiers (HEKA Electronik, Germany), P-97 Flaming/Brown type micropipette puller (Sutter Instrument Co, USA), Masterflex L/S peristaltic pump (Cole-Parmer Instrument, Malaysia), AxioCam ICm 1 photo-video camera (Carl Zeiss, Germany), borosilicate glass capillaries GC120F-7.5 (Harvard apparatus, UK), the software for recording and processing the electrophysiological data - PatchMaster (HEKA Electronik, Germany), MiniAnalysis (Synaptosoft Inc., USA). 
To measure neuronal activity, there was used an extracellular solution containing (mmol) $130 \mathrm{NaCl}$; $2.5 \mathrm{KCl} ; 1.5 \mathrm{MgCl}_{2} ; 1.5 \mathrm{CaCl}_{2} ; 10$ glucose; 10 HEPES at $24^{\circ} \mathrm{C}$, osmolarity $-300 \pm 5 \mathrm{mOsm}$; pH 7.3-7.4; and intracellular solution (mmol): 130 K-gluconate; 10 HEPES; 2 EGTA; 3 L-ascorbic acid; $2 \quad \mathrm{MgCl}_{2}$; $1 \mathrm{Na}_{2}$-GTP; $2 \quad \mathrm{Na}_{2}$-ATP (pH 7.2; osmolarity - 295 $3 \mathrm{mOsm}$ ). A similar intracellular solution with QX-314 bromide at $2 \mathrm{mM}$ concentration was used to block sodium currents when recording spontaneous excitatory postsynaptic currents.

The study involved cells with access resistance below $20 \mathrm{MOhm}$ and leakage current of $100 \ldots 100 \mathrm{pA}$. The resting potential for neurons was maintained at $70 \mathrm{mV}$. The following parameters of action potential were studied: amplitude (calculated based on the points from the subthreshold potential to the overshoot point); hyperpolarization amplitude (the difference between the lower point of hyperpolarization period after the action potential and the baseline of stimulation step where this potential was generated); threshold potential amplitude (calculated based on the rate of potential increase for each cell). The above parameters were determined based on the first action potential on the threshold stimulus. Spontaneous neuronal activity was recorded in the cell culture for $5 \mathrm{~min}$. Membrane capacity was tested by applying $5 \mathrm{mV}$ pulses and calculated based on changes in current.

GraphPad Prism 6 (GraphPad Software Inc., USA) was used for making statistical analysis and building the graphs.

Multi-electrode recording of neural network activity. The neural network of the brain is known to be a structural and functional unit responsible for processing, storage, and retrieval of information. For the multi-electrode recording of network activity in vitro, primary cultures of brain cells (hippocampal cells, in particular) were grown directly on multi-electrode arrays. The spontaneous bioelectric activity was recorded using the arrays of the MEA60 multi-electrode system (Multichannel Systems, Germany) under standard environmental conditions: temperature, humidity, carbon dioxide and oxygen content [14].

To obtain the data, we used the MC Rack software (Multichannel Systems, Germany). The main features of spontaneous bioelectric activity of the neural network were investigated: frequency and duration of small network bursts, the number of spikes in a burst, interburst interval duration, percentage of free spikes.

Electron microscopy. For electron microscopic studies, the tissue was treated using the standard technique. A $2.5 \%$ solution of glutaraldehyde on a phosphate buffer $(\mathrm{pH} 7.4)$ and a $1 \%$ solution of osmium tetraoxide with potassium ferricyanide were used for fixation, sequentially. The tissue was dehydrated in alcohols of ascending concentration (to 100\% alcohol), then - in 100\% acetone. After dehydration, the tissue was treated with a mixture $(1: 1)$ of $100 \%$ acetone and a mixture of Epon-Araldite resins for $1 \mathrm{~h}$. Next, the tissue was embedded in a mixture of Epon-Araldite for one more hour followed by resin polarization. Ultrathin sections were cut on the Leica EMT UC7 ultramicrotome (Leica Microsystems, Germany). They were contrasted with uranyl acetate and Reynolds' lead citrate, then examined using Morgagni 268D electron microscope (FIE, USA). Synaptic contacts were counted in five fields of vision with an area of $1600 \mu \mathrm{m}^{2}$.

Statistics. The obtained data on the changes in Oregon Green 488 BAPTA-1 AM dye fluorescence, reflecting the dynamics of intracellular calcium (frequency and duration of calcium events), morphometric and electrophysiological data were presented as mean \pm standard error of the mean $(M \pm S E M)$. The significance of statistical differences in samples was tested using nonparametric Mann-Whitney U-test. To identify statistically significant differences in the features of bioelectric activity, ANOVA analysis for one parameter and the Student-Newman-Kells method were used. The differences between samples were considered statistically significant at $p<0.05$.

\section{Results and Discussion}

Studies of metabolic activity based on short-term changes in intracellular calcium concentration allowed us to evaluate such characteristics of neuron-glia network functions as the duration and frequency of calcium events (Figure 1). Carbamylated darbepoetin had no significant effect on the frequency of calcium oscillations in neurons $(4.88 \pm 0.12 \mathrm{~s}$ in the control group and $3.87 \pm 0.57 \mathrm{~s}$ in the experimental group) and glia $(0.74 \pm 0.07$ and $0.57 \pm 0.17 \mathrm{~s}$, respectively), or the average duration of calcium events in neurons $(5.6 \pm 1.05 \mathrm{~s}$ in the control group and $7.17 \pm 1.59 \mathrm{~s}$ in the experimental group) and glial cells $(11.7 \pm 0.85$ and $10.75 \pm 0.11 \mathrm{~s}$, respectively).

Electrophysiological studies showed no statistically significant changes in the following action potential parameters under the influence of CdEpo: the size $(92.10 \pm 1.42 \mathrm{mV}$ in the control and $91.98 \pm 3.30 \mathrm{mV}$ in the experimental group) (Figure 2 (d)); hyperpolarization value $(-4.75 \pm 0.33 \mathrm{mV}$ in the control and $-4.38 \pm 0.59 \mathrm{mV}$ in the experimental group) (Figure 2 (c)); the value of threshold potential required for action potential emergence $(7.24 \pm 1.57 \mathrm{mV}$ in the control and 4.62 $\pm 1.04 \mathrm{mV}$ during incubation with CdEpo) (Figure 2 (b)). Membrane capacity exhibited no changes after treatment of cultures with CdEpo either $(1.132 \pm 0.1330 \mathrm{nF}$ in the control and $1.209 \pm 0.3371 \mathrm{nF}$ in the experimental group) (Figure 2 (a)). There were no statistically significant changes in the frequency of spontaneous action potential emergence $(p=0.2398)$.

Studies of neural network activity revealed no statistically significant differences in primary hippocampal cultures treated with carbamylated darbepoetin (Figure 3). The parameters of spikes in a 


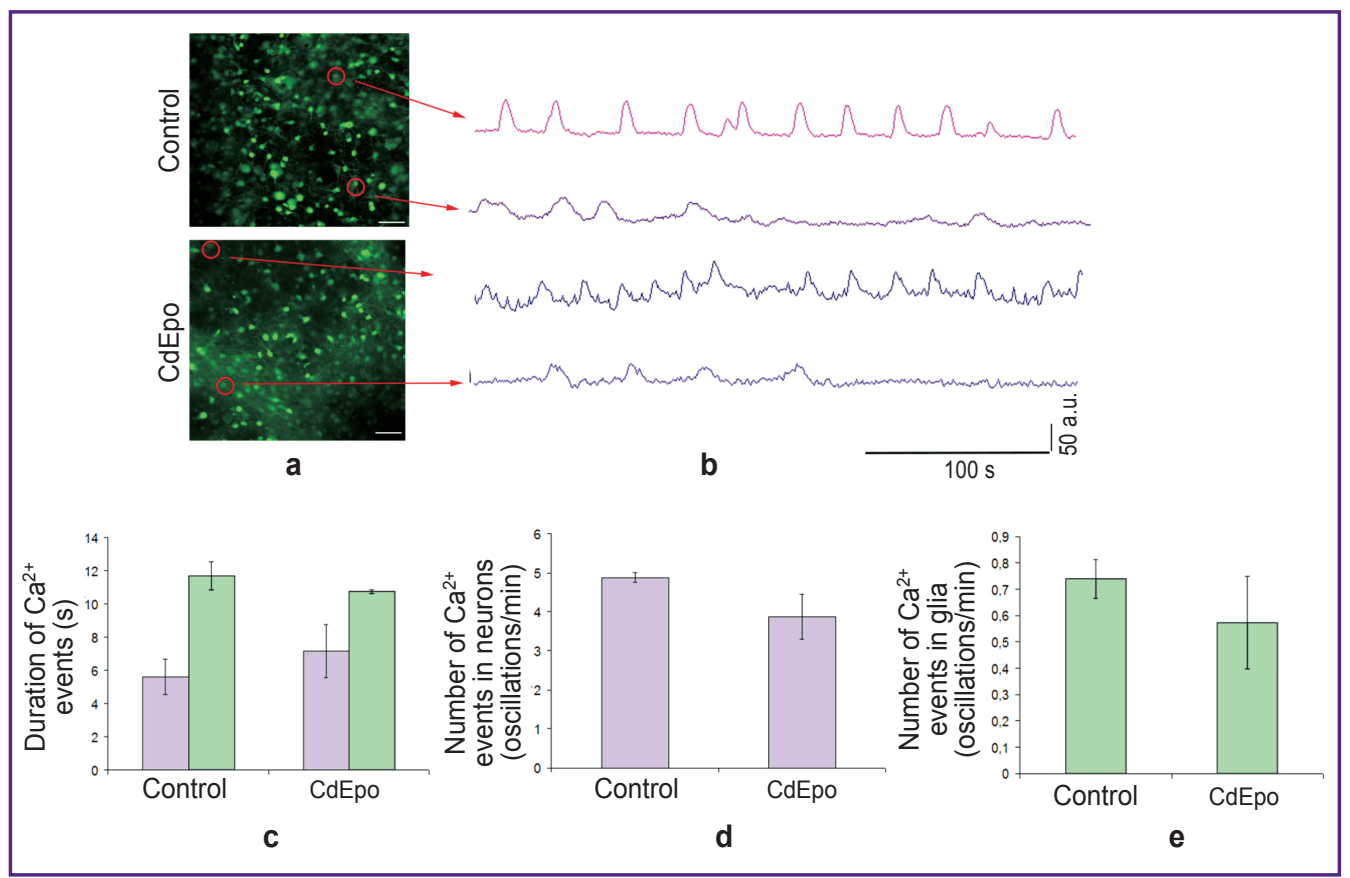

Figure 1. The influence of CdEpo on calcium activity characteristics:

(a) fluorescent images of dissociated cells stained with Oregon Green 488 BAPTA-1 AM; (b) example curves of time-dependent changes in absolute fluorescence intensity of Oregon Green 488 BAPTA-1 AM in the control and experimental groups; (c) the average duration of single calcium oscillations; (d) the average frequency of single calcium oscillations in neurons; (e) the average frequency of single calcium oscillations in glia

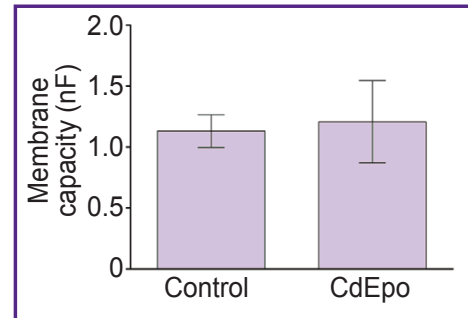

a
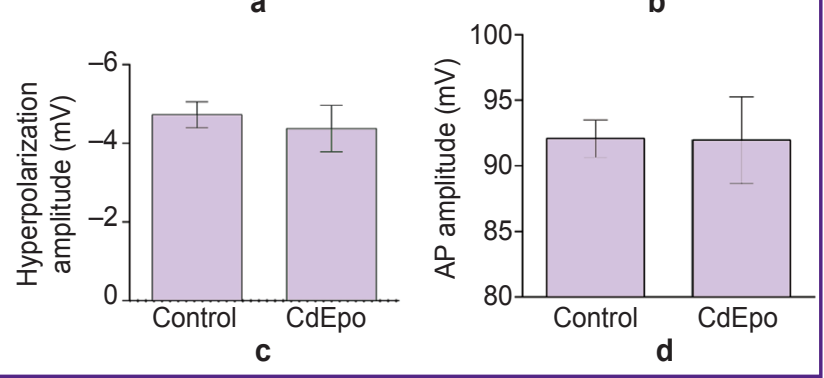

Figure 2. Electrophysiological parameters of neurons after $24 \mathrm{~h}$ incubation of cells with CdEpo $(100 \mathrm{ng} / \mathrm{ml})$ :

(a) membrane capacity; (b) threshold potential (TP) amplitude; (c) hyperpolarization amplitude; (d) action potential (AP) amplitude

burst (Figure $3(\mathrm{a}),(\mathrm{c}))$ and the characteristics of spike bursts themselves (Figure 3 (b), (d), (e)) remained unchanged as well as the percentage of free spikes of neurons uninvolved in neural network activity (Figure $3(\mathrm{f})$ ).

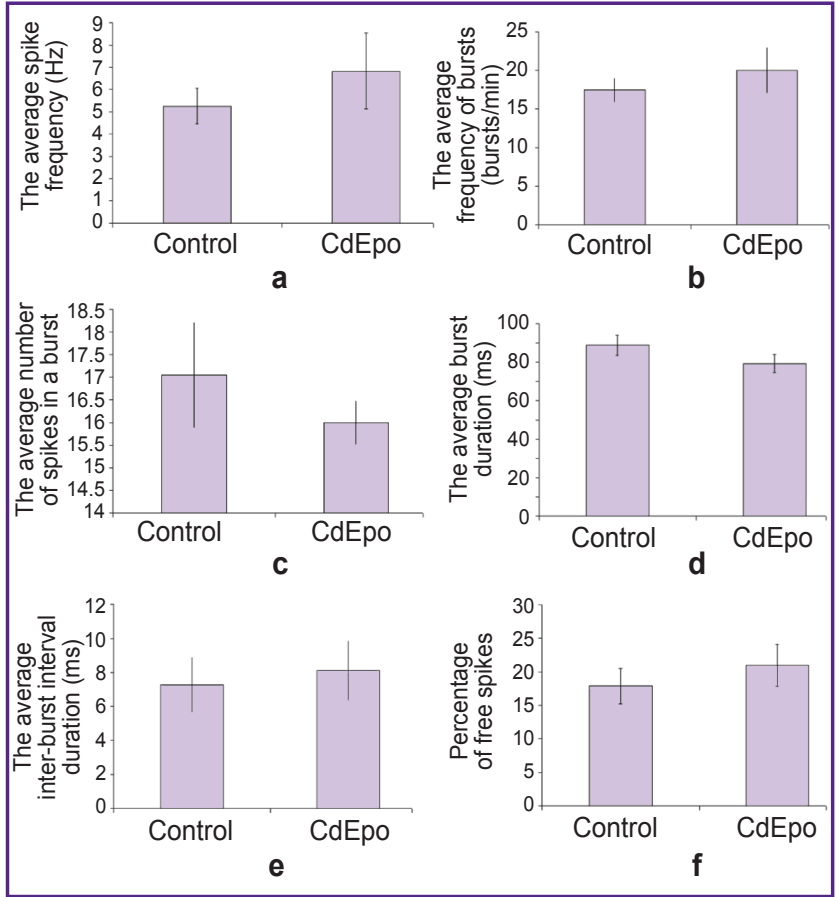

Figure 3. The influence of CdEpo $(50 \mathrm{ng} / \mathrm{ml})$ on the bioelectric parameters of neural network activity of dissociated hippocampal cell cultures:

(a) spike frequency; (b) the frequency of bursts; (c) the number of spikes in a burst; (d) the duration of bursts; (e) inter-burst interval duration; (f) free spikes (not included in bursts) in the inter-burst interval 
Ultrastructural analysis of the synaptic pool of neural networks in primary hippocampal cultures revealed no statistically significant differences in the number of mature chemical asymmetric contacts between groups (Figure 4 (a)). However, there was an increase in the number of dendritic spines with endoplasmic reticulum and the spine apparatus (Figure 4 (b), Figure 5 (f)) rarely found in primary cultures of mouse hippocampal cells. The qualitative analysis of the ultrastructure of intracellular organelles (mitochondria, nuclei) showed no significant changes in the ultrastructural organization. Cristae of mitochondria were well visualized in the cells of both control (Figure 5 (a)) and experimental groups (Figure 5 (d)). Osmiophilic glycogen granules and various organelles with the intact structure were observed in glial cells (Figure 5 (b) and (e)).

Thus, investigation of CdEpo effects on intact neuron-glia networks after $24 \mathrm{~h}$ in vitro demonstrated the absence of neurotrophic effect of carbamylated

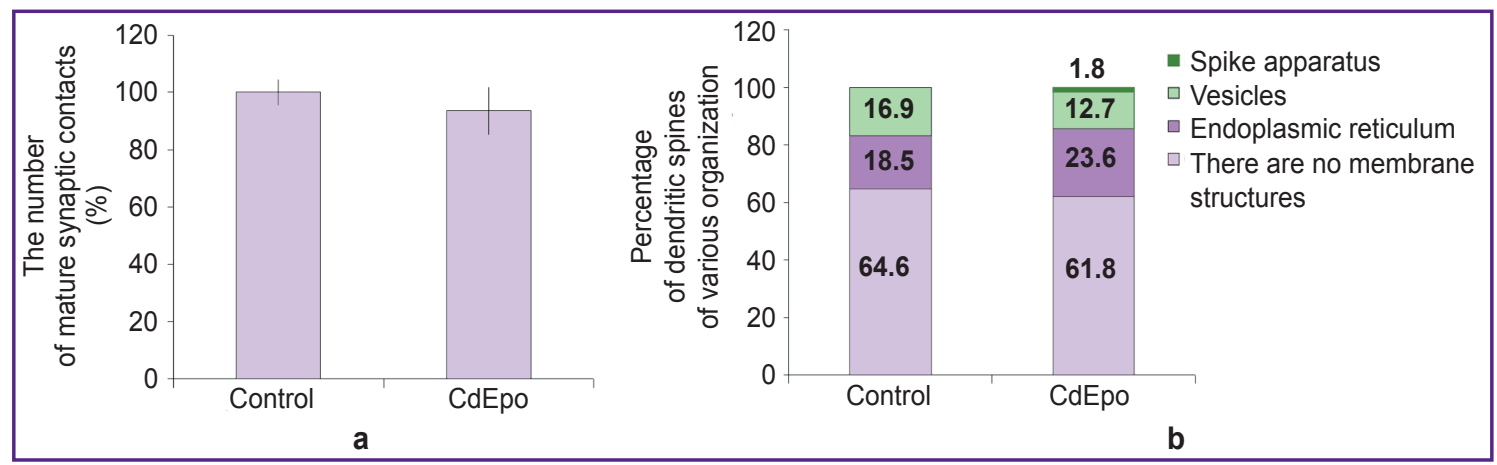

Figure 4. Characteristics of synaptic contacts in neural networks of primary hippocampal cultures and the proportion of dendritic spines with the various intracellular organization after $24 \mathrm{~h}$ of cell incubation with CdEpo (100 ng/ml):

(a) the number of mature asymmetric chemical synapses; (b) percentage of dendritic spines with the various internal organization in the control and experimental groups

Figure 5. Representative electron microscopy images of neuron-glia network cells after their $24 \mathrm{~h}$ incubation with CdEpo (100 $\mathrm{ng} / \mathrm{ml})$ :

control: (a) neuron cell body; (b) accumulation of mitochondria in the process; (c) perforated asymmetric synapse; CdEpo: (d) neuron cell body; (e) the dendrite of the neuron and astrocyte cell body; (f) the spine apparatus in the dendritic spine; the arrow indicates synaptic contact. Here: ER - endoplasmic reticulum, A - axon, GI glial cell, D - dendrite, DS - dendritic spine, GC - growth cone, M - mitochondria, SA spine apparatus, $\mathrm{N}-$ nucleus

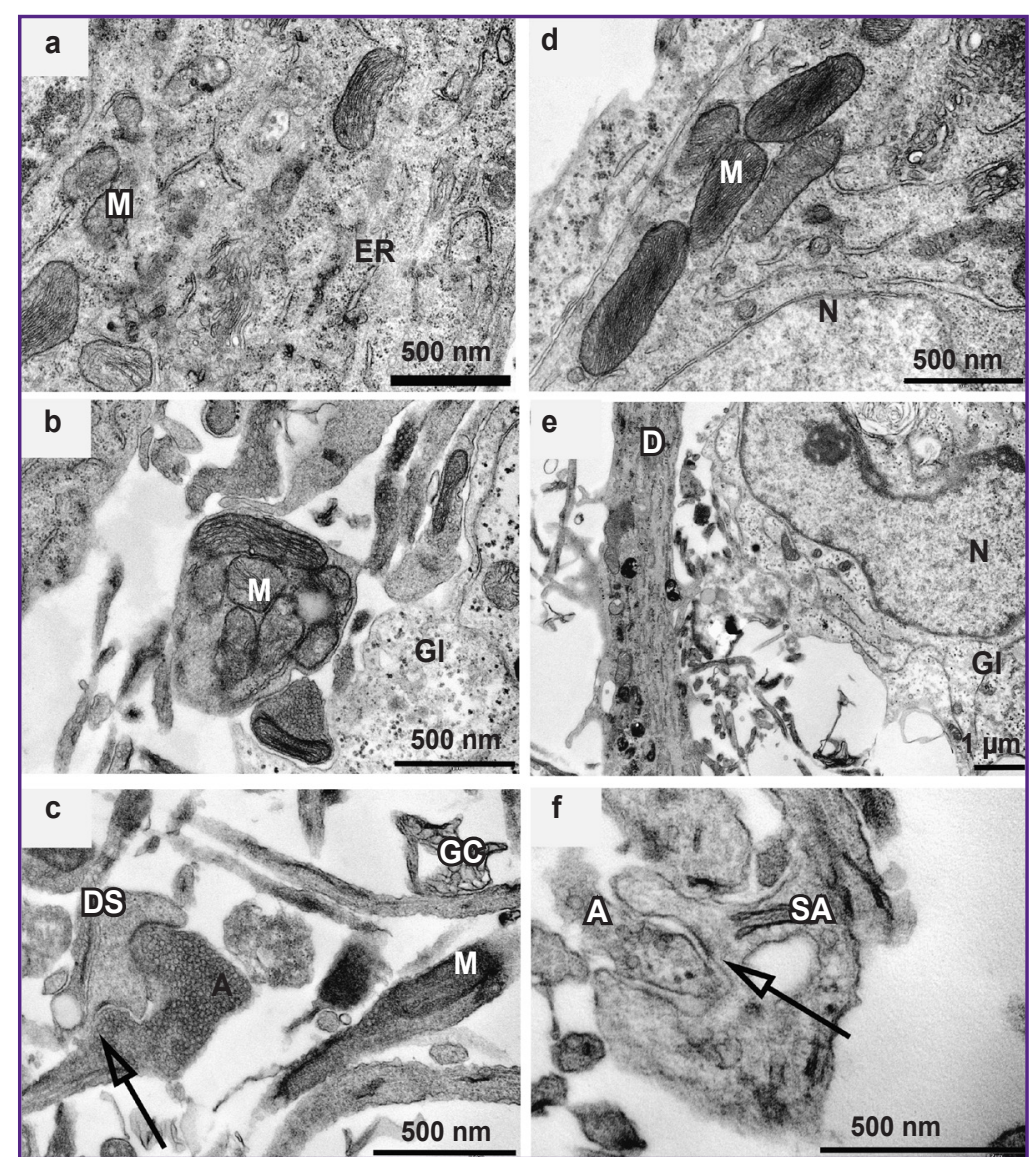


erythropoietin derivative. Therefore, activation of cerebral erythropoietin receptors produced no changes in neuronal excitability parameters, particularly, potential-dependent ion channels involved in action potential generation. Moreover, after CdEpo application in normoxia, there was no change in astrocyte activity based on intracellular calcium registration parameters as a characteristic of spontaneous activity of non-excitable glial cells.

The absence of neurotrophic effect of CdEpo on the model of primary hippocampal culture proves the hypothesis that erythropoietin is primarily a cytoprotector triggering intracellular signaling cascade [15] contributing to cellular adaptation to hypoxia, which underlies the improvement in neuronal and glial functions as observed in experimental modeling of ischemia/hypoxia.

Indeed, our studies have revealed the adaptogenic effect of CdEpo on spike apparatus morphogenesis even in normoxia, which allows modulating the signal coming to the cell through the synapse. It was shown earlier [16] that the spine apparatus is likely to influence the fast and slow components of calcium oscillation damping ensured by calcium pumps present in the organelle and the physical presence of the spine apparatus in the spine neck (isolation effect).

\section{Conclusion}

Carbamylated darbepoetin produces no significant effects on the functions of intact neurons and glia, which is demonstrated by the absence of statistically significant differences in action potential parameters and duration of calcium events in single cells, the absence of changes in bioelectric network activity or damage to the ultrastructure of dissociated hippocampal cells after $24 \mathrm{~h}$ treatment with CdEpo. Therefore, it is relatively safe to use this molecule as a cytoprotector for therapeutic purposes.

Study funding. The study was supported by the Russian Foundation for Basic Research within the framework of scientific project No.18-34-00877.

Conflict of interests. The authors have no conflict of interest to disclose.

\section{References}

1. Hernández C.C., Burgos C.F., Gajardo A.H., Silva-Grecchi T., Gavilan J., Toledo J.R., Fuentealba J. Neuroprotective effects of erythropoietin on neurodegenerative and ischemic brain diseases: the role of erythropoietin receptor. Neural Regen Res 2017; 12(9): 1381-1389, https:// doi.org/10.4103/1673-5374.215240.

2. Ponce L.L., Navarro J.C., Ahmed O., Robertson C.S. Erythropoietin neuroprotection with traumatic brain injury. Pathophysiology 2013; 20(1): 31-38, https://doi.org/10.1016/j. pathophys.2012.02.005.

3. Alnaeeli M., Wang L., Piknova B., Rogers H., Li X.,
Noguchi C.T. Erythropoietin in brain development and beyond. Anat Res Int 2012; 2012: 953264, https://doi. org/10.1155/2012/953264.

4. Chavez J.C., Baranova O., Lin J., Pichiule P. The transcriptional activator hypoxia inducible factor 2 (HIF2/EPAS-1) regulates the oxygen-dependent expression of erythropoietin in cortical astrocytes. J Neurosci 2006; 26(37): 9471-9481, https://doi.org/10.1523/jneurosci.283806.2006 .

5. Khan A.I., Coldewey S.M., Patel N.S., Rogazzo M., Collino M., Yaqoob M.M., Radermacher P., Kapoor A., Thiemermann C. Erythropoietin attenuates cardiac dysfunction in experimental sepsis in mice via activation of the $\beta$-common receptor. Dis Model Mech 2013; 6(4): 1021-1030, https://doi. org/10.1242/dmm.011908.

6. Leist M., Ghezzi P., Grasso G., Bianchi R., Villa P., Fratelli M., Savino C., Bianchi M., Nielsen J., Gerwien J., Kallunki P., Larsen A.K., Helboe L., Christensen S., Pedersen L.O., Nielsen M., Torup L., Sager T., Sfacteria A., Erbayraktar S., Erbayraktar Z., Gokmen N., Yilmaz O., Cerami-Hand C., Xie Q.W., Coleman T., Cerami A., Brines M. Derivatives of erythropoietin that are tissue protective but not erythropoietic. Science 2004; 305(5681): 239-242, https://doi. org/10.1126/science.1098313.

7. Brines M., Cerami A. Emerging biological roles for erythropoietin in the nervous system. Nat Rev Neurosci 2005; 6(6): 484-494, https://doi.org/10.1038/nrn1687.

8. Bohr S., Patel S.J., Vasko R., Shen K., IrachetaVellve A., Lee J., Bale S.S., Chakraborty N., Brines M., Cerami A., Berthiaume F., Yarmush M.L. Modulation of cellular stress response via the erythropoietin/CD131 heteroreceptor complex in mouse mesenchymal-derived cells. J Mol Med 2015; 93(2): 199-210, https://doi.org/10.1007/s00109-0141218-2.

9. Elliott S., Busse L., Bass M.B., Lu H., Sarosi I., Sinclair A.M., Spahr C., Um M., Van G., Begley C.G. Anti-Epo receptor antibodies do not predict Epo receptor expression. Blood 2006; 107(5): 1892-1895, https://doi.org/10.1182/ blood-2005-10-4066.

10. Kirkeby A., van Beek J., Nielsen J., Leist M., Helboe L. Functional and immunochemical characterisation of different antibodies against the erythropoietin receptor. J Neurosci Methods 2007; 164(1): 50-58, https://doi.org/10.1016/j. jneumeth.2007.03.026.

11. Zhuchenko M.A., Serebryakova M.V., Seregin Yu.A., Cherepushkin S.A., Lobanova N.V., Klishin A.A., Vologzhannikova A.A., Kazakov A.S., Permyakov S.E. Carbamylated darbepoetin alfa: structure and properties. Biotekhnologiya 2017; 33(4): 28-43, https://doi. org/10.21519/0234-2758-2017-33-4-28-43.

12. Shirokova O.M., Frumkina L.E., Vedunova M.V., Mitroshina E.V., Zakharov Y.N., Khaspekov L.G., Mukhina I.V. Morphofunctional patterns of neuronal network developing in dissociated hippocampal cell cultures. Sovremennye tehnologii $v$ medicine 2013; 5(2): 6-13.

13. Zakharov Y.N., Mitroshina E.V., Vedunova M.V., Korotchenko S.A., Kalintseva Y.I., Mukhina I.V., Potanina A.V. Fluorescence analysis of the metabolic activity patterns of a neuronal-glial network. Journal of Optical Technology 2012; 79(6): 348, https://doi.org/10.1364/jot.79.000348.

14. Pimashkin A., Gladkov A., Mukhina I., Kazantsev V. Adaptive enhancement of learning protocol in hippocampal cultured networks grown on multielectrode arrays. Front Neural 
Circuits 2013; 7, https://doi.org/10.3389/fncir.2013.00087. 15. Ding J., Wang J., Li Q.Y., Yu J.Z., Ma C.G., Wang X., Lu C.Z., Xiao B.G., Neuroprotection and CD131/GDNF/AKT pathway of carbamylated erythropoietin in hypoxic neurons. Mol Neurobiol 2017; 54(7): 5051-5060, https://doi.org/10.1007/ s12035-016-0022-0.
16. Majewska A., Brown E., Ross J., Yuste R. Mechanisms of calcium decay kinetics in hippocampal spines: role of spine calcium pumps and calcium diffusion through the spine neck in biochemical compartmentalization. J Neurosci 2000; 20(5): 1722-1734, https://doi.org/10.1523/ jneurosci.20-05-01722.2000. 